Proyecciones Journal of Mathematics

Vol. 29, No 3, pp. 181-191, December 2010.

Universidad Católica del Norte

Antofagasta - Chile

\title{
A NEW DEFINITION OF $S^{*}$ CLOSEDNESS IN $L-T O P O L O G I C A L$ SPACES
}

\author{
BO CHEN \\ SOUTHWEST UNIVERSITY, P. R. CHINA \\ Received : June 2009. Accepted : May 2010
}

\begin{abstract}
In this paper, a new notion of $S^{*}$ closedness in L-topological Spaces is introduced by means of semi-open $L$-sets and their inequality where $L$ is a complete DeMorgan algebra.This new definition doesn't rely on the structure of basic lattice L. It can be characterized by means of semi-open L-sets and their inequality. When $L$ is completely distributive DeMorgan algebra, its many characterizations are presented.
\end{abstract}

Keywords and Phrases : semiopen $L-$ set, $S^{*}$ closedness, $L$-topological space.

Mathematics Subject Classification(2000) : 54A40,54A20. 


\section{Introduction}

Compactness is one of the most important notions in topology. In [1], Azad introduced the concepts of semiopen sets and semiopen continuous functions in $L-$ topology. In [5], Kudri generalized the concept of semicompactness and $S^{*}$ closedness in $L$ - topological spaces. However, Kudri's $S^{*}$ closedness relies on the structure of $L$ which is a completely distributive lattice.

In [10], Shi introduced a new definition of fuzzy compactness in $L-$ topological spaces by means of open $L-$ sets and their inequality where $L$ is a completely distributive DeMorgan algebra. This new definition doesn't rely on the structure of basic lattice $L$.

In this paper, along the line of [10] we shall introduce a new definition of $S^{*}$ closedness in $L$ - topological spaces by means of semiopen $L$ - sets and their inequality. Also we give characterizations and properties of $S^{*}$ closedness .

\section{Preliminaries}

Throughout this paper, $\left(L, \bigvee, \Lambda^{\prime},\right)$ is a complete DeMorgan algebra, $X$ is a nonempty set. $L^{X}$ is the set of all $L$ - fuzzy sets ( $L$-sets for short) on $X$. The smallest element and the largest element in $L^{X}$ are denoted by $\underline{0}$ and $\underline{1}$.

An element $a$ in $L$ is called prime element if $a \geq b \wedge c$ implies $a \geq b$ or $a \geq c$. $a$ in $L$ is called co-prime element if $a^{\prime}$ is a prime element. The set of non-unit prime elements in $L$ is denoted by $P(L)$. The set of non-zero co-prime elements in $L$ is denoted by $M(L)$.

The binary relation $\prec$ in $L$ is defined as follows: for $a, b \in L, a \prec b$ if and only if for every subset $D \subset L$, the relation $b \leq \sup D$ always implies the existence of $d \in D$ with $a \leq d$. In a complete distributive DeMorgan algebra $L$, each element $b$ is a sup of $\{a \in L \mid a \prec b\},\{a \in L \mid a \prec b\}$ is called the greatest minimal family of $b$ in the sense of [16], in symbol $\beta(b)$. Moreover for $b \in L$, define $\beta^{*}(b)=\beta(b) \bigcap M(L), \alpha(b)=\left\{a \in L \mid a^{\prime} \prec b^{\prime}\right\}$ and $\alpha^{*}(b)=\alpha(b) \bigcap P(L)$.

For $a \in L$ and $A \in L^{X}$, let

$$
\begin{gathered}
A_{[a]}=\{x \in X \mid A(x) \geq a\}, \\
A_{(a)}=\{x \in X \mid a \in \beta(A(x))\}, \\
A^{(a)}=\{x \in X \mid A(x) \not \leq a\} .
\end{gathered}
$$


An $L$-topological space ( $L$ - space for short) is a pair $(X, \delta)$, where $\delta$ is a subfamily of $L^{X}$ which contains $\underline{0} \underline{1}$ and is closed for any suprema and infima. $\delta$ is called an $L$-topology on $X$. Every member of $\delta$ is called an open $L$-set and its quasicomplementation is called a closed $L$-set.

If $(X, \tau)$ is a topological space, then $\omega(\tau)$ denote the $L$-topology of the continuous functions from $(X, \tau)$ to $L$ with its $\operatorname{Scott}$ topology. $(X, \omega(\tau))$ is called to be topologically generated by $(X, \delta)$.

For a subfamily $\Phi \subseteq L^{X}, 2^{(\Phi)}$ denotes the set of all finite subfamily of $\Phi$.

Definition $2.1([1,9])$ Let $(X, \delta)$ be an $L$-space. $A \in L^{X}$ is called semiopen if and only if there exists $B \in \delta$ such that $B \leq A \leq \operatorname{cl}(B)$. $A \in L^{X}$ is called semiclosed if and only if $A^{\prime}$ is semiopen.

Definition 2.2([1,9]) Let $(X, \delta)$ be an $L$-space, $A \in L^{X}$. The set $c l_{*} A=$ $\bigwedge\left\{B \in L^{X} \mid A \leq B\right.$ and $B$ is semiclosed $\}$ is called the semiclosure of $A$. int $_{*} A=\bigvee\left\{B \in L^{X} \mid B \leq A\right.$ and $B$ is semiopen $\}$ is called the semiinterior of $A$.

Definition 2.3([10]) Let $(X, \delta)$ be an $L$-space. $G \in L^{X}$ is called compact if for every family $\mathcal{U}$ of open $L$ - sets, it follows that

$$
\bigwedge_{x \in X}\left(G^{\prime}(x) \vee \bigvee_{A \in \mathcal{U}} A(x)\right) \leq \bigvee_{\mathcal{V} \in \in(\mathcal{U})} \bigwedge_{x \in X}\left(G^{\prime}(x) \vee \bigvee_{A \in \mathcal{V}} A(x)\right)
$$

Lemma 2.4([10]) Let $L$ be complete Heyting algebra and let $f:\left(X, \delta_{1}\right) \rightarrow$ $\left(Y, \delta_{2}\right)$ be a mapping. Then for any family $\mathcal{P} \subset L^{Y}$

$$
\bigvee_{y \in Y}\left(f(G)(y) \wedge \bigwedge_{B \in \mathcal{P}} B(y)\right)=\bigvee_{x \in X}\left(G(x) \wedge \bigwedge_{B \in \mathcal{P}} f^{-1}(B)(x)\right)
$$

Definition 2.5([10,11]) Let $(X, \delta)$ be an $L$-space, $a \in L \backslash\{1\}$, and $G \in$ $L^{X}$. A family $\mathcal{U} \subset L^{X}$ is said to be

(1) an $a$-shading of $G$ if for any $x \in X,\left(G^{\prime}(x) \vee \bigvee_{A \in \mathcal{U}} A(x)\right) \not \leq a$;

(2) a strong $a$-shading of $G$ if $\bigwedge_{x \in X}\left(G^{\prime}(x) \vee \bigvee_{A \in \mathcal{U}} A(x)\right) \not \leq a$;

(3) an $a$ - remote family $\mathcal{P}$ of $G$ if for any $x \in X,\left(G(x) \wedge \bigwedge_{A \in \mathcal{U}} B(x)\right) \nsupseteq a$;

(4) a strong $a$ - remote family of $G$ if $\bigvee_{x \in X}\left(G(x) \wedge \bigwedge_{A \in \mathcal{U}} A(x)\right) \nsupseteq a$.

Definition 2.6([10]) Let $a \in L \backslash\{0\}, G \in L^{X}$. A family $\mathcal{U} \subset L^{X}$ is called 
(1) a $\beta_{a}-$ cover of $G$ if for all $x \in X$, it follows that $a \in \beta\left(G^{\prime}(x) \vee\right.$ $\left.\bigvee_{A \in \mathcal{U}} A(x)\right)$

(2) a strong $\beta_{a}-$ cover of $G$ if $a \in \beta\left(\bigwedge_{x \in X}\left(G^{\prime}(x) \vee \bigvee_{A \in \mathcal{U}} A(x)\right)\right)$;

(3) a $Q_{a}-$ cover of $G$ if for all $x \in X$, it follows that $G^{\prime}(x) \vee \bigvee_{A \in \mathcal{U}} A(x) \geq$ $a$

Definition 2.7 $([1,9])$ Let $\left(X, \delta_{1}\right)$ and $\left(Y, \delta_{2}\right)$ be two $L$-spaces. $f:\left(X, \delta_{1}\right) \rightarrow$ $\left(Y, \delta_{2}\right)$ is called

(1) semicontinuous if $f^{-1}(A)$ is semiopen in $\left(X, \delta_{1}\right)$ for every open $L-$ set $A$ in $\left(Y, \delta_{2}\right)$.

(2) irresolute if $f^{-1}(A)$ is semiopen in $\left(X, \delta_{1}\right)$ for every semiopen $L-$ set $A$ in $\left(Y, \delta_{2}\right)$.

\section{Definition and characterizations of $S^{*}$ closedness}

Definition 3.1 Let $(X, \delta)$ be an $L$-space. $G \in L^{X}$ is called $S^{*}$ closedness if for every family $\mathcal{U}$ of semiopen $L$ - sets, it follows that

$$
\bigwedge_{x \in X}\left(G^{\prime}(x) \vee \bigvee_{A \in \mathcal{U}} A(x)\right) \leq \bigvee_{\mathcal{V} \in \in^{(\mathcal{U})}} \bigwedge_{x \in X}\left(G^{\prime}(x) \vee \bigvee_{A \in \mathcal{V}} c l_{*} A(x)\right)
$$

Example 3.2 Suppose $X=\{x\}$, and $L=\{0,1 / 4,1 / 2,3 / 4,1\}$. For $a \in L$, $a^{\prime}=1-a$. Let $\delta=\{\underline{0}, A, \underline{1}\}$, where $A(x)=3 / 4$, then $(X, \delta)$ is an $L$-space. We can see that every $L$-set in $(X, \delta)$ is $S^{*}$ closedness.

Since any open $L$-set must be semiopen $L$ - set,we have the following theorem.

Theorem 3.3 Compactness is $S^{*}$ closedness in $L$-space.

From Definition 3.1 and quasicomplementation of $L$, we can obtain the following theorem.

Theorem 3.4 Let $(X, \delta)$ be an $L$-space. $G \in L^{X}$ is $S^{*}$ closedness if and only for every family $\mathcal{B}$ of semiclosed $L$ - sets, it follows that

$$
\bigvee_{x \in X}\left(G(x) \wedge \bigwedge_{B \in \mathcal{B}} B(x)\right) \geq \bigwedge_{\mathcal{F} \in \bigwedge^{(\mathcal{B})}} \bigvee_{x \in X}\left(G(x) \wedge \bigwedge_{B \in \mathcal{F}} i n t_{*} B(x)\right)
$$

Definition 3.5 Let $a \in L \backslash\{0\}, G \in L^{X}$. A family $\mathcal{U} \subset L^{X}$ is said to have weak $a$-nonempty intersection in $G$ if $\bigvee_{x \in X}\left(G(x) \wedge \bigwedge_{A \in \mathcal{U}} A(x)\right) \geq a$. $\mathcal{U}$ is said to have finite weak $a_{*}$-intersection property in $G$ if for every finite 
subfamily $\mathcal{F}$ of $\mathcal{U}$, the family $\mathcal{F}_{*}=\left\{i n t_{*} F \mid F \in \mathcal{F}\right\}$ has weak $a$-nonempty intersection in $G$.

Theorem 3.6 Let $(X, \delta)$ be an $L$-space, $G \in L^{X}$. Then the following conditions are equivalent:

(1) $G$ is $S^{*}$ closedness.

(2) For any $a \in L \backslash\{1\}$, each semiopen strong $a$ - shading $\mathcal{U}$ of $G$ has a finite subfamily $\mathcal{V}$ such that $\left\{c l_{*} A \mid A \in \mathcal{V}\right\}$ is a strong $a$ - shading of $G$.

(3) For any $a \in L \backslash\{0\}$, each semiclosed strong $a$ - remote family $\mathcal{P}$ of $G$ has a finite subfamily $\mathcal{F}$ such that $\left\{\right.$ int $\left._{*} A \mid A \in \mathcal{P}\right\}$ is a strong $a$ - remote family of $G$.

(4) For any $a \in L \backslash\{0\}$, each family of semiclosed $L$-sets which has the finite weak $a_{*}$-intersection property in $G$ has weak $a$-nonempty intersection in $G$.

Proof The proofs follow from Definition 3.1 and Theorem 3.4.

\section{Properties of $S^{*}$ closedness}

Theorem 4.1 Let $L$ be complete Heyting algebra. If $G$ and $H$ are $S^{*}$ closedness, then so is $G \bigvee H$.

Proof For any family $\mathcal{P}$ of of semiclosed $L$ - sets, by Theorem 3.4 we have that

$$
\begin{aligned}
\bigvee_{x \in X} & \left((G \bigvee H)(x) \wedge \bigwedge_{B \in \mathcal{P}} B(x)\right) \\
& =\left\{\bigvee_{x \in X}\left(G(x) \wedge \bigwedge_{B \in \mathcal{P}} B(x)\right)\right\} \bigvee\left\{\bigvee_{x \in X}\left(H(x) \wedge \bigwedge_{B \in \mathcal{P}} B(x)\right)\right\} \\
& \geq\left\{\bigwedge_{\mathcal{F} \in \in^{(\mathcal{P})}} \bigvee_{x \in X}\left(G(x) \wedge \bigwedge_{B \in \mathcal{F}} i n t_{*} B(x)\right)\right\} \bigvee\left\{\bigwedge_{\mathcal{F} \in \in(\mathcal{P})} \bigvee_{x \in X}\left(H(x) \wedge \bigwedge_{B \in \mathcal{F}} i n t_{*} B(x)\right)\right\} \\
& =\bigwedge_{\mathcal{F} \in \in^{(\mathcal{P})}} \bigvee_{x \in X}\left((G \bigvee H)(x) \wedge \bigwedge_{B \in \mathcal{F}} i n t_{*} B(x)\right)
\end{aligned}
$$

This shows that $G \bigvee H$ is $S^{*}$ closedness.

Theorem 4.2 If $G$ is $S^{*}$ closedness and $H$ is a semiclosed set, then $G \wedge H$ is $S^{*}$ closedness.

Proof For any family $\mathcal{P}$ of of semiclosed $L$ - sets, by Theorem 3.4 we have that 


$$
\begin{aligned}
\bigvee_{x \in X} & \left((G \bigwedge H)(x) \wedge \bigwedge_{B \in \mathcal{P}} B(x)\right) \\
& =\bigvee_{x \in X}\left(G(x) \wedge \bigwedge_{B \in \mathcal{P} \bigcup\{\mathcal{H}\}} B(x)\right) \\
\geq & \left\{\bigwedge_{\left.\mathcal{F} \in \in^{(\mathcal{P}} \bigcup\{\mathcal{H}\}\right)} \bigvee_{x \in X}\left(G(x) \wedge \bigwedge_{B \in \mathcal{F}} i n t_{*} B(x)\right)\right\} \\
= & \left\{\bigwedge_{\mathcal{F} \in \in(\mathcal{P})} \bigvee_{x \in X}\left(G(x) \wedge \bigwedge_{B \in \mathcal{F}} i n t_{*} B(x)\right)\right\} \\
& \bigvee\left\{\bigwedge_{\mathcal{F} \in \in^{(\mathcal{P})}} \bigvee_{x \in X}\left(G(x) \bigwedge H(x) \wedge \bigwedge_{B \in \mathcal{F}} i n t_{*} B(x)\right)\right\} \\
= & \bigwedge_{\mathcal{F} \in \in(\mathcal{P})} \bigvee_{x \in X}\left((G \bigwedge H)(x) \wedge \bigwedge_{B \in \mathcal{F}} i n t_{*} B(x)\right)
\end{aligned}
$$

Then we obtain that $G \wedge H$ is $S^{*}$ closedness.

Theorem 4.3 Let $L$ be complete Heyting algebra and $f:\left(X, \delta_{1}\right) \rightarrow\left(Y, \delta_{2}\right)$ be an irresolute mapping. If $G$ is $S^{*}$ closedness in $\left(X, \delta_{1}\right)$, then $f(G)$ is $S^{*}$ closedness in $\left(Y, \delta_{2}\right)$.

Proof For any family $\mathcal{P}$ of of semiclosed $L$ - sets in $\left(Y, \delta_{2}\right)$, by Lemma 2.4 and $S^{*}$ closedness of $G$ we have that

$$
\begin{aligned}
\bigvee_{y \in Y} & \left((f(G))(y) \wedge \bigwedge_{B \in \mathcal{P}} B(y)\right) \\
& =\bigvee_{x \in X}\left(G(x) \wedge \bigwedge_{B \in \mathcal{P}} f^{-1}(B)(x)\right) \\
& \geq \bigwedge_{\mathcal{F} \in \in(\mathcal{P})} \bigvee_{x \in X}\left(G(x) \wedge \bigwedge_{B \in \mathcal{F}} i n t_{*} f^{-1}(B)(x)\right) \\
& \geq \bigwedge_{\mathcal{F} \in \in(\mathcal{P})} \bigvee_{x \in X}\left(G(x) \wedge \bigwedge_{B \in \mathcal{F}} f^{-1}\left(i n t_{*} B\right)(x)\right) \\
& =\bigwedge_{\mathcal{F} \in \in(\mathcal{P})} \bigvee_{y \in Y}\left(f(G)(y) \wedge \bigwedge_{B \in \mathcal{F}} i n t_{*} B(y)\right)
\end{aligned}
$$

Hence $f(G)$ is $S^{*}$ closedness.

Analogously, we can obtain the following theorem.

Theorem 4.4 Let $L$ be complete Heyting algebra and $f:\left(X, \delta_{1}\right) \rightarrow\left(Y, \delta_{2}\right)$ be a semicontinous mapping. If $G$ is $S^{*}$ closedness in $\left(X, \delta_{1}\right)$, then $f(G)$ is $S^{*}$ closedness in $\left(Y, \delta_{2}\right)$. 


\section{Further characterizations of $S^{*}$ closedness and goodness}

In this section, we assume that $L$ is a completely distributive DeMorgan algebra.

Theorem 5.1 Let $(X, \delta)$ be an $L$-space, $G \in L^{X}$. Then the following statements are equivalent:

(1) $G$ is $S^{*}$ closedness.

(2) For any $a \in L \backslash\{0\}$, each semiclosed strong $a$ - remote family $\mathcal{P}$ of $G$ has a finite subfamily $\mathcal{F}$ such that $\left\{\operatorname{int}_{*} A \mid A \in \mathcal{F}\right\}$ is a strong $a$ - remote family of $G$.

(3) For any $a \in L \backslash\{0\}$, each semiclosed strong $a$ - remote family $\mathcal{P}$ of $G$ has a finite subfamily $\mathcal{F}$ such that $\left\{\operatorname{int}_{*} A \mid A \in \mathcal{F}\right\}$ is an $a$ - remote family of $G$.

(4) For any $a \in L \backslash\{0\}$, each semiclosed strong $a$ - remote family $\mathcal{P}$ of $G$ there exist a finite subfamily $\mathcal{F}$ of $\mathcal{P}$ and $b \in \beta(a)$ such that $\left\{\right.$ int $\left._{*} A \mid A \in \mathcal{F}\right\}$ is a strong $b$ - remote family of $G$.

(5) For any $a \in L \backslash\{0\}$, each semiclosed strong $a$ - remote family $\mathcal{P}$ of $G$ there exist a finite subfamily $\mathcal{F}$ of $\mathcal{P}$ and $b \in \beta(a)$ such that $\left\{\right.$ int $\left._{*} A \mid A \in \mathcal{F}\right\}$ is a $b$ - remote family of $G$.

(6) For any $a \in L \backslash\{1\}$, each semiopen strong $a-\operatorname{shading} \mathcal{U}$ of $G$ has a finite subfamily $\mathcal{V}$ such that $\left\{c l_{*} A \mid A \in \mathcal{V}\right\}$ is a strong $a$ - shading of $G$.

(7) For any $a \in L \backslash\{1\}$, each semiopen strong $a$ - shading $\mathcal{U}$ of $G$ has a finite subfamily $\mathcal{V}$ such that $\left\{c l_{*} A \mid A \in \mathcal{V}\right\}$ is an $a$ - shading of $G$.

(8) For any $a \in L \backslash\{1\}$ and any semiopen strong $a$ - shading $\mathcal{U}$ of $G$ ,there exist a finite subfamily $\mathcal{V}$ and $b \in \alpha(a)$ such that $\left\{c l_{*} A \mid A \in \mathcal{V}\right\}$ is a $b$ - shading of $G$.

(9) For any $a \in L \backslash\{1\}$ and any semiopen strong $a$ - shading $\mathcal{U}$ of $G$ ,there exist a finite subfamily $\mathcal{V}$ and $b \in \alpha(a)$ such that $\left\{c l_{*} A \mid A \in \mathcal{V}\right\}$ is a strong $b$ - shading of $G$.

(10) For any $a \in L \backslash\{0\}$, each semiopen strong $\beta_{a}$-cover $\mathcal{U}$ of $G$ has a finite subfamily $\mathcal{V}$ such that $\left\{c l_{*} A \mid A \in \mathcal{V}\right\}$ is a strong $\beta_{a}$-cover of $G$.

(11) For any $a \in L \backslash\{0\}$, each semiopen strong $\beta_{a}$-cover $\mathcal{U}$ of $G$ has a finite subfamily $\mathcal{V}$ such that $\left\{c_{*} A \mid A \in \mathcal{V}\right\}$ is a $\beta_{a}$-cover of $G$.

(12) For any $a \in L \backslash\{0\}$ and any semiopen strong $\beta_{a}$-cover $\mathcal{U}$ of $G$, there exist a finite subfamily $\mathcal{V}$ and $b \in L$ with $a \in \beta(b)$ such that $\left\{c l_{*} A \mid A \in \mathcal{V}\right\}$ is a strong $\beta_{a}$-cover of $G$.

(13) For any $a \in L \backslash\{0\}$ and any semiopen strong $\beta_{a}$-cover $\mathcal{U}$ of $G$, there exist a finite subfamily $\mathcal{V}$ and $b \in L$ with $a \in \beta(b)$ such that $\left\{c l_{*} A \mid A \in \mathcal{V}\right\}$ is a $\beta_{a}$-cover of $G$. 
(14) For any $a \in L \backslash\{0\}$ and $b \in \beta(a) \backslash\{0\}$,each semiopen $Q_{a}$-cover $\mathcal{U}$ of $G$ has a finite subfamily $\mathcal{V}$ such that $\left\{c l_{*} A \mid A \in \mathcal{V}\right\}$ is a $Q_{b}$-cover of $G$.

(15) For any $a \in L \backslash\{0\}$ and $b \in \beta(a) \backslash\{0\}$, each semiopen $Q_{a}$-cover $\mathcal{U}$ of $G$ has a finite subfamily $\mathcal{V}$ such that $\left\{c l_{*} A \mid A \in \mathcal{V}\right\}$ is a strong $\beta_{b}-$ cover of $G$.

(16) For any $a \in L \backslash\{0\}$ and $b \in \beta(a) \backslash\{0\}$, each semiopen $Q_{a}$-cover $\mathcal{U}$ of $G$ has a finite subfamily $\mathcal{V}$ such that $\left\{c l_{*} A \mid A \in \mathcal{V}\right\}$ is a $\beta_{b}$-cover of $G$.

Proof $(1) \Leftrightarrow(2)$ It follows from Theorem 3.6.

$(2) \Rightarrow(3)$ It is easy for the fact that every strong $a$ - remote family of $G$ is $a$ - remote family of $G$.

$(3) \Rightarrow(4)$ Let $a \in L \backslash\{0\}$. Suppose $\mathcal{U}$ is semiclosed strong $a-$ remote family of $G$, then $\bigvee_{x \in X}\left(G(x) \wedge \bigwedge_{A \in \mathcal{U}} A(x)\right) \nsupseteq a$. Take $c \in \beta(a)$ such that $\bigvee_{x \in X}\left(G(x) \wedge \bigwedge_{A \in \mathcal{U}} A(x)\right) \geq c$. One can see that $\mathcal{U}$ is semiclosed strong $c$ - remote family of $G$. From $(3), \mathcal{U}$ has a finite subfamily $\mathcal{F}$ such that $\left\{\right.$ int $\left._{*} A \mid A \in \mathcal{F}\right\}$ is $c$ - remote family of $G$. Choose $b \in \beta(a)$ such that $c \in \beta(b)$, then $\left\{\operatorname{int}_{*} A \mid A \in \mathcal{F}\right\}$ is strong $b$ - remote family of $G$.

$(4) \Rightarrow(5) \Rightarrow(2)$ It is Obvious.

$(1) \Leftrightarrow(6)$ It follows from Theorem 3.6.

$(6) \Rightarrow(7)$ It is easy for the fact that every strong $a$-shading family of $G$ is $a$-shading family of $G$.

(7) $\Rightarrow(8)$ Let $a \in L \backslash\{1\}$. Suppose $\mathcal{U}$ is semiclosed strong $a$ - shading family of $G$, then $\bigwedge_{x \in X}\left(G^{\prime}(x) \vee \bigvee_{A \in \mathcal{U}} A(x)\right) \not z a$. Take $c \in \alpha(a)$ such that $\bigwedge_{x \in X}\left(G^{\prime}(x) \vee \bigvee_{A \in \mathcal{U}} A(x)\right) \not \leq c$. One can see that $\mathcal{U}$ is semiclosed strong $c$-shading family of $G$. From $(7), \mathcal{U}$ has a finite subfamily $\mathcal{F}$ such that $\left\{\right.$ int $\left._{*} A \mid A \in \mathcal{F}\right\}$ is $c$-shading family of $G$. Choose $b \in \alpha(a)$ such that $c \in \alpha(b)$, then $\left\{\right.$ int $\left._{*} A \mid A \in \mathcal{F}\right\}$ is strong $b$-shading family of $G$.

$(8) \Rightarrow(9) \Rightarrow(6)$ It is Obvious.

Similarly we can prove the other statements.

Remark 5.2 In Theorem 5.1, $a \in L \backslash\{0\}$ and $b \in \beta(a)$ can be replaced by $a \in M(L)$ and $b \in \beta^{*}(a)$ respectively. $a \in L \backslash\{1\}$ and $b \in \alpha(a)$ can be replaced by $a \in P(L)$ and $b \in \alpha^{*}(a)$ respectively. Thus, we can obtain other 15 equivalent conditions of the $S^{*}$ closedness.

Lemma 5.3([11]) Let $(X, \omega(\delta))$ be generated topologically by $(X, \delta)$. If $A$ is a semiopen set in $(X, \delta)$, then $\chi_{A}$ is a semiopen set $L$ - set in $(X, \omega(\delta))$. If $B$ is a semiopen set in $(X, \omega(\delta))$, then $B_{(a)}$ is a semiopen set in $(X, \delta)$. 
Lemma 5.4 Let $(X, \omega(\delta))$ be generated topologically by $(X, \delta), B \in L^{X}$. Then $c l_{*}\left(B_{[b]}\right) \subset\left(c l_{*} B\right)_{[b]}$ in $(X, \omega(\delta))$.

Proof For $B \in L^{X}, B_{[b]} \subset\left(c l_{*} B\right)_{[b]}$. Then $c l_{*}\left(B_{[b]}\right) \subset\left(c l_{*} B\right)_{[b]}$ in $(X, \omega(\delta))$ since $\left(c l_{*} B\right)_{[b]}$ is a semiclosed set in $(X, \omega(\delta))$ and $c l_{*}\left(B_{[b]}\right)$ is the smallest semiclosed set contains $B_{[b]}$.

Theorem 5.5 Let $(X, \delta)$ be a topological space and $(X, \omega(\delta))$ be generated topologically by $(X, \delta)$. Then $(X, \omega(\delta))$ is $S^{*}$ closedness if and only if $(X, \delta)$ is $S^{*}$ closedness.

Proof Let $\mathcal{A}$ be a semiopen-cover of $(X, \delta)$. Then $\left\{\chi_{A} \mid A \in \mathcal{A}\right\}$ is a family of semiopen $L-$ sets in $(X, \omega(\delta))$ with $\bigwedge_{x \in X}\left(\bigvee_{A \in \mathcal{U}} \chi_{A}(x)\right)=\underline{1}$. From the $S^{*}$ closedness of $(X, \omega(\delta))$, it follows that

$$
\underline{1}=\bigvee_{\mathcal{V} \in \in(\mathcal{U})} \bigwedge_{x \in X}\left(\bigvee_{A \in \mathcal{V}} c l_{*}\left(\chi_{A}\right)(x)\right) \leq \bigvee_{\mathcal{V} \in \in(\mathcal{U})} \bigwedge_{x \in X}\left(\bigvee_{A \in \mathcal{V}} \chi_{c l_{*} A}(x)\right)
$$

This implies that there exists $\mathcal{V} \in \in^{(\mathcal{U})}$ such that $\bigvee_{A \in \mathcal{V}} \chi_{c l_{*} A}(x)=\underline{1}$. Hence, the family of $\left\{c l_{*}(A) \mid A \in \mathcal{V}\right\}$ is a cover of $(X, \delta)$. Then $(X, \delta)$ is $S^{*}$ closedness.

Conversely, let $\mathcal{U}$ be a family of semiopen $L-$ sets in $(X, \omega(\delta))$ and let $\bigwedge_{x \in X}\left(\bigvee_{B \in \mathcal{U}} B(x)\right)=a$. If $a=0$, then obviously

$$
\bigwedge_{x \in X}\left(\bigvee_{B \in \mathcal{U}} B(x)\right) \leq \bigvee_{\mathcal{V} \in \in(\mathcal{U})} \bigwedge_{x \in X}\left(\bigvee_{B \in \mathcal{V}} c l_{*} B(x)\right)
$$

Now we suppose that $a \neq 0$. In this case, for any $b \in \beta(a) \backslash\{0\}$, we have that

$$
b \in \beta\left(\bigwedge_{x \in X}\left(\bigvee_{B \in \mathcal{U}} B(x)\right)\right) \subset \bigcap_{x \in X} \beta\left(\bigvee_{B \in \mathcal{U}} B(x)\right)=\bigcap_{x \in X} \bigcup_{B \in \mathcal{U}} \beta(B(x))
$$

This implies the family of $\left\{B_{(b)} \mid B \in \mathcal{U}\right\}$ is a semiopen cover of $(X, \delta)$ by Lemma 5.3 , From the $S^{*}$ closedness of $(X, \delta)$, there exists $\mathcal{V} \in \in^{(\mathcal{U})}$ such that $\left\{c l_{*}\left(B_{(b)}\right) \mid B \in \mathcal{V}\right\}$ is a cover of $(X, \delta)$. Obviously $\left\{\left(c l_{*} B\right)_{[b]} \mid B \in \mathcal{V}\right\}$ is a cover of $(X, \delta)$ since $c l_{*}\left(B_{(b)}\right) \subset c l_{*}\left(B_{[b]}\right) \subset\left(c l_{*} B\right)_{[b]}$. Hence $b \leq$ $\bigwedge_{x \in X}\left(\bigvee_{B \in \mathcal{V}} c l_{*} B(x)\right)$. Further we have that

$$
b \leq \bigwedge_{x \in X}\left(\bigvee_{B \in \mathcal{V}} c l_{*} B(x)\right) \leq \bigvee_{\mathcal{V} \in \in^{(\mathcal{U})}} \bigwedge_{x \in X}\left(\bigvee_{B \in \mathcal{V}} c l_{*} B(x)\right)
$$

This implies that

$$
\bigwedge_{x \in X}\left(\bigvee_{B \in \mathcal{U}} B(x)\right)=a=\bigvee\{b \mid b \in \beta(a)\} \leq \bigvee_{\mathcal{V} \in \in(\mathcal{U})} \bigwedge_{x \in X}\left(\bigvee_{B \in \mathcal{V}} c l_{*} B(x)\right)
$$


Therefore $(X, \omega(\delta))$ is $S^{*}$ closedness.

Corollary 5.6 $S^{*}$ closedness in $L$-topological spaces is good extension.

\section{References}

[1] K. K. Azad, On fuzzy semicontinuity,fuzzy almost continuity and fuzzy weakly continuity, J. Math. Anal. Appl, 82, pp. 14-32, (1981).

[2] C. L. Chang, Fuzzy topological spaces, J. Math. Anal. Appl, 24, pp. 182-190, (1968).

[3] B. Ghosh, Semicontinuous and semiclosed mappings and semiconnectedness in fuzzy setting, Fuzzy Sets and Systems, 35, pp. 345355 , (1990).

[4] G. Gierz, et al., A compendium of continuous Lattices, Springer Verlag, Berlin,1980.

[5] S. R. T. Kudri, Semicompactness and $S^{*}$-closedness in $L$-fuzzy topological spaces, Fuzzy Sets and Systems, 109, pp. 223-231, (2000).

[6] S. R. T. Kudri, M. W. Warner, Some good L-fuzzy compactnessrelated concepts and their properties I, Fuzzy Sets and Systems, 76, pp. 141-155, (1995).

[7] S. R. T. Kudri, M. W. Warner, Some good $L$-fuzzy compactnessrelated concepts and their properties II, Fuzzy Sets and Systems, 76, pp. 157-168, (1995).

[8] Y. M. Liu, M. K. Luo, Fuzzy topology,World Scientific,Singapore, (1997).

[9] S. Malakar, On fuzzy semi-irresolute and strongly irresolute functions, Fuzzy Sets and Systems, 45, pp. 239-244, (1992).

[10] F. G. Shi, A new notion of fuzzy compactness in $L$-topological spaces, Information Science. 173, pp. 35-48, (2005).

[11] F. G. Shi, Semicompactness in $L$-topological spaces, Internatinal Journal of Mathematics and Mathematical Sciences, 12, pp. 1869-1878, (2005). 
[12] F. G. Shi, A new form of fuzzy $\beta$-compactness, Proyecciones Journal of Mathematics, 24, pp. 105-119, (2005).

[13] F. G. Shi, Countable compactness and the Lindelöf property of $L$-fuzzy sets, Iranian Journal of Fuzzy System, 1, pp. 79-88, (2004).

[14] F. G. Shi, A new approach to fuzzy almost compactness, Proyecciones Journal of Mathematics, 28, pp. 75-87, (2009).

[15] F. G. Shi, A new form of fuzzy $\alpha$-compactness, Mathematica Bohemica 131, pp. 15-28, (2006).

[16] G. J. Wang, Theory of $L$-fuzzy topological spaces,Shaanxi Normal University Press, Xi'an, (1988).

\section{Bo Chen}

School of Mathematics and Statistics

Southwest University

Chongqing 400715

P. R. China

e-mail : bobochen@swu.edu.cn 